\title{
Analytical Approach to Continuous and Intermittent Bottleneck Flows
}

\author{
Dirk Helbing and Anders Johansson \\ Dresden University of Technology, Andreas-Schubert-Strasse 23, 01062 Dresden, Germany \\ Joachim Mathiesen, Mogens H. Jensen, and Alex Hansen \\ Department of Physics, Norwegian University of Science and Technology, 7491 Trondheim, Norway, \\ and The Niels Bohr Institute, Blegdamsvej 17, 2100 Copenhagen, Denmark
}

(Received 16 May 2006; published 17 October 2006)

\begin{abstract}
We propose a many-particle-inspired theory for granular outflows from a hopper and for the escape dynamics through a bottleneck based on a continuity equation in polar coordinates. If the inflow is below the maximum outflow, we find an asymptotic stationary solution. If the inflow is above this value, we observe queue formation, which can be described by a shock wave equation. We also address the experimental observation of intermittent outflows, taking into account the lack of space in the merging zone by a minimum function and coordination problems by a stochastic variable. This results in avalanches of different sizes even if friction, force networks, inelastic collapse, or delay-induced stop-and-go waves are not assumed. Our intermittent flows result from a random alternation between particle propagation and gap propagation. Erratic flows in congested merging zones of vehicle traffic may be explained in a similar way.
\end{abstract}

PACS numbers: $45.70 . \mathrm{Vn}$

Driven granular media display a rich spectrum of pattern formation phenomena. This includes collective oscillating states, convection patterns, the spontaneous segregation of different granular materials, and the formation of avalanches due to self-organized criticality [1]. Here, we will focus on jamming and clogging phenomena [2] related to arching [3] and on intermittent outflows through hoppers [4,5]. Similar phenomena are known from dense pedestrian crowds [6]. The escape dynamics of individuals from a room has been intensively studied, showing that in crowd stampedes, rooms are emptied in an irregular, strongly intermittent fashion [7]. This effect has been discovered in simulations performed with the social and the centrifugal force model $[7,8]$, with cellular automata and lattice gas automata [9], and in a mean-field model [10]. It has also been experimentally confirmed $[6,11]$. However, analytical models of escape dynamics and granular bottleneck flows are lacking.

In this Letter we will formulate such a model. Our goal is to gain a better understanding of (i) the resulting density profiles and (ii) the irregular outflows at bottlenecks. The model not only addresses the distribution of the avalanche sizes in the outflow from a bottleneck, but it also offers a possible explanation of the long-standing problem of perturbations forming in merging zones of freeway traffic flows $[12,13]$, which are characterized by erratic, forward or backward moving shock waves [12]. It is believed that these can trigger stop-and-go waves in traffic flows $[12,14]$. Similar findings have been made in overcrowded pedestrian flows [11] and expected for merging flows in urban traffic and production networks.

In all these cases, the competition of too many entities for little space leads to coordination problems. We are therefore looking for a minimal, common model capturing this feature. Hence, we will first abstract from specific system features such as the non-Newtonian character of real granular flows, nonslip boundary conditions, dissipative interactions, or force networks in quasistatic granular flows $[15,16]$, and discuss extensions later. This will allow us to show that intermittent flows are caused even without mechanisms like dissipative collapse [15], large spatiotemporal fluctuations due to force networks [16], or delayinduced instabilities (as in traffic flows). These may magnify the effect [17].

As pedestrian evacuation has been successfully described by driven granular particle models, where a single particle represents an individual pedestrian, we will formulate a common model for escaping pedestrians and gravity-driven outflows from vertical, two-dimensional hoppers. Because of the conservation of the particle number, we will describe the aggregate, two-dimensional particle flow by the continuity equation for the particle density $\rho$ as a function of space and time. Both the shape of a funnel and the semicircular shape of a waiting crowd suggest to write this equation in polar coordinates. Assuming no significant dependence on the polar angles $\theta$ and $\varphi$ for the moment, we obtain $\partial \rho / \partial t+(1 / r) \partial(r \rho v) / \partial r=0$ (generalizing this to a $2 d$ treatment later). Here, $t$ denotes the time, $r \geq 0$ the distance from the bottleneck (exit), and $v \leq 0$ the velocity component in radial direction. The above continuity equation can be rewritten as

$$
\frac{\partial \rho}{\partial t}+\frac{\partial(\rho v)}{\partial r}=-\frac{\rho v}{r}
$$

where the term on the right-hand side reflects a merging effect similar to an on-ramp or lane closure term in a model of freeway traffic. By use of logarithmic derivatives, the above equation can be rewritten as $\partial \ln \rho(r, t) / \partial t=$ $-v(r, t) \partial \ln [r \rho(r, t) v(r, t)] / \partial r$. For the stationary case with $\partial \ln \rho / \partial t=0$ it follows from $\partial \ln (r \rho v) / \partial r=0$ that 
the overall flow $f \pi r \rho(r) v(r)$ through any cross section at distance $r$ is constant:

$$
f \pi r \rho(r) v(r)=f \pi r q(r)=:-Q_{0}=\text { const. }
$$

$q(r)=\rho(r) v(r)$ is the particle flow through a cross section of unit length. $f=1$ corresponds to the half circumference $\pi r$ of a circle of radius $r$, while $f<1$ allows one to treat hoppers with an opening angle smaller than $180^{\circ}$. (The walls should be steeper than the angle of repose.) $Q_{0} \geq 0$ is the stationary overall particle flow.

To facilitate the derivation of analytical results, we will assume the linear velocity-density relationship

$$
v(r)=V(\rho(r))=-v_{0}\left(1-\rho / \rho_{\max }\right) \leq 0 .
$$

$v_{0}$ means the maximum ("free") particle speed and $\rho_{\max }$ the maximum particle density. Equations (2) and (3) give the quadratic equation $\rho(r) v_{0}\left[1-\rho(r) / \rho_{\max }\right]=$ $Q_{0} /(f \pi r)$ in $\rho$. With $r_{\text {crit }}\left(Q_{0}\right):=Q_{0} /\left(f \pi q_{\max }\right)$ it implies

$$
\rho_{ \pm}\left(r, Q_{0}\right)=\frac{\rho_{\max }}{2}\left(1 \pm \sqrt{\left.1-\frac{r_{\text {crit }}\left(\overline{Q_{0}}\right)}{r}\right)},\right.
$$

where $q_{\max }=v_{0} \rho_{\max } / 4$ is the maximum flow. In free flow with $d|\rho V(\rho)| / d \rho \geq 0$, the density profile is determined by the upstream boundary condition, i.e., $Q_{0}$ is given by the overall inflow $Q_{\text {in }}$. Under congested conditions $[d|\rho V(\rho)| / d \rho<0], Q_{0}$ is given by the overall outflow $Q_{\text {out }}=\min \left(Q_{\text {in }}, 2 r_{0} q_{\max }, f \pi r_{0} q_{\max }\right)$, i.e., the minimum of the overall inflow $Q_{\text {in }}$ and the maximum possible overall outflow. The stationary case requires $Q_{\text {in }}=Q_{\text {out }}$ and a non-negative discriminant in Eq. (4). This calls for $r_{0} \geq r_{\text {crit }}\left(Q_{\text {in }}\right)$, i.e., large outlets [see Fig. 1(a)]. Then, $\rho(r, t)$ converges to a stationary free flow with the density profile $\rho_{-}\left(r, Q_{\text {in }}\right) \leq \rho_{\max } / 2$. The density profile for other velocity-density relationships than (3) can be obtained numerically. Smooth perturbations like the humps in Fig. 1(a) propagate forward at the speed $V(\rho)+\rho d V(\rho) / d \rho=-v_{0}\left(1-2 \rho / \rho_{\max }\right) \quad[18]$, compactify close to the outlet, and leave the system.

If the overall inflow exceeds the overall outflow $\left(Q_{\text {in }}>\right.$ $Q_{\text {out }}$ ), particles are jammed behind the outlet [Fig. 1(b)]. The initial density profile $\rho(r, 0)$ again approaches $\rho_{-}\left(r, Q_{\text {in }}\right)<\rho_{\max } / 2$ in the free-flow regime at large distances $r$, but converges to $\rho_{+}\left(r, Q_{\text {out }}\right)>\rho_{\max } / 2$ in the congested regime upstream of the outlet. The congestion front moves forward until the jam density $\rho_{+}\left(r, Q_{\text {out }}\right)$ is reached. Then, the particles queue up and the shock front at location $R(t)$ moves backward at the speed

$$
\frac{d R}{d t}=-\frac{Q_{\text {in }}-Q_{\text {out }}}{f \pi R(t)\left[\rho_{-}\left(R, Q_{\text {in }}\right)-\rho_{+}\left(R, Q_{\text {out }}\right)\right]}
$$

according to the shock wave equation [18] [see Fig. 1(b)]. Hence, we find the free-flow density profile $\rho(r, t) \approx$ $\rho_{-}\left(r, Q_{\text {in }}\right)$ for $r>R(t)$, while for $r<R(t)$ we have the congested density profile $\rho(r, t) \approx \rho_{+}\left(r, Q_{\text {out }}\right)$.

This applies to cases of continuous outflows, which are observed for large enough openings $[2,8]$ or small enough

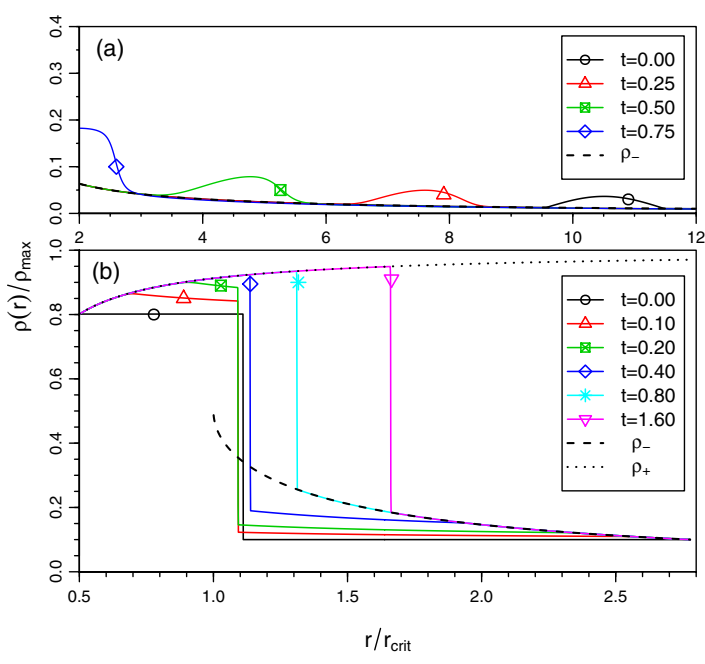

FIG. 1 (color online). Density profiles at different times: (a) when the inflow is low and the initial density profile has a hump, (b) the inflow exceeds the maximum outflow and the initial density profile is a step function (shock wave). The simulation results have been obtained by solving the continuity equation with the Godunov scheme, assuming $\rho\left(12 r_{\text {crit }}, t\right)=$ 0.01 and floating boundary conditions at $r=r_{0}=2 r_{\text {crit }}:=$ $2 r_{\text {crit }}\left(Q_{\text {in }}\right)$ in case (a), but $\rho\left(2.8 r_{\text {crit }}, t\right)=0.1$ and $Q_{\text {out }}=$ $2 r_{0} q_{\max }$ (corresponding to the maximum outflow) with $r_{0}=$ $0.5 r_{\text {crit }}$ in case (b). Note that the asymptotic density profile is $\rho_{-}\left(r, Q_{\text {in }}\right)$ in free flow and $\rho_{+}\left(r, Q_{\text {out }}\right)$ in jammed flow.

pedestrian velocities $v_{0}$ [7]. However, if the desired velocity $v_{0}$ of pedestrians is high, their maximum density $\rho_{\max }$ goes up and intermittent outflows are observed $[7,11]$. This intermittent behavior (see Fig. 2) reminds one of driven granular media [4] and shall be modeled now. For this, let us subdivide the particle bulk into shells of thickness $\Delta r$ (for example, the particle diameter $d$ or multiples of it). Within each shell of area $A(r) \approx f \pi r \Delta r$, we assume a constant average density $\rho(r, t)=N(r, t) / A(r)$, where $N(r, t)$ denotes the number of particles in the shell of radius $r$ at time $t$. Furthermore, we assume that particles move from one shell to the next with velocity $v_{0}$, if they find a suitable gap; otherwise, they will stay. The maximum number of particles available to move into the shell of radius $r$ is $\rho(r+\Delta r, t) A(r+\Delta r)$, while the maximum number of available gaps in shell $r$ is $\rho_{\max } A(r) \times$ $\left[1-\rho(r, t) / \rho_{\max }\right]$, because $\rho_{\max } A(r)$ is the maximum number of particles in the shell of radius $r$ and $q(r, t)=$ $1-\rho(r, t) / \rho_{\max }$ represents the fraction of free space. Finally, we assume that $\xi_{r}^{ \pm} q(r, t)$ denotes the probability to find a suitable gap in front of a particle allowing it to move, where $\xi_{r}^{ \pm}$are random numbers specified in each time step with $0 \leq \xi_{r}^{ \pm} \leq 1$ and $\xi_{r-\Delta r}^{+}=\xi_{r}^{-}$(in order to guarantee particle conservation). Then, the number of inflowing particles within the time interval $\Delta t=\Delta r / v_{0}$ is $N_{\text {in }}(r, t)=\xi_{r}^{+} q(r, t) \min \left[A(r+\Delta r) \rho(r+\Delta r, t), A(r) \rho_{\max }\right]$, while the number of outflowing particles is $N_{\text {out }}(r, t)=$ $\xi_{r}^{-} q(r-\Delta r, t) \min \left[A(r) \rho(r, t), A(r-\Delta r) \rho_{\text {max }}\right]$. From 


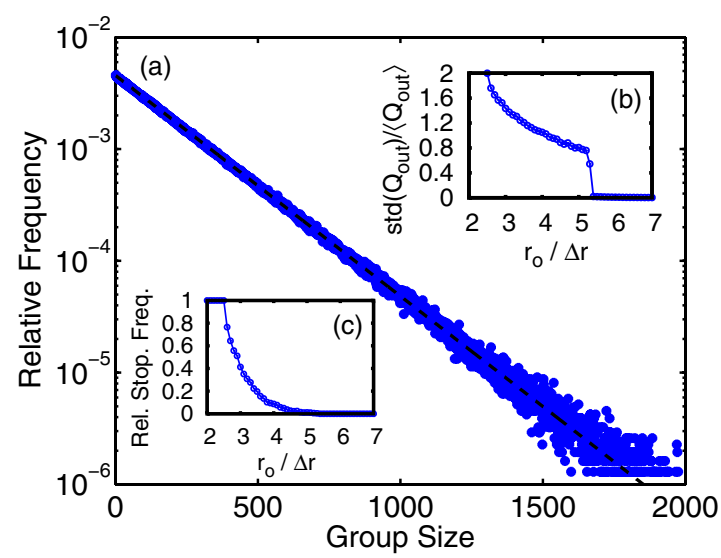

FIG. 2 (color online). (a) In agreement with an experiment for granular outflows from a two-dimensional hopper [5], our simulation model generates exponentially distributed avalanche sizes when frictionless particles with coordination problems are jamming at a bottleneck (i.e., theory and experiment show a straight line in a log-linear plot). (b) The standard deviation of the outflow, divided by the average outflow shows 3 regimes: no outflow for $r_{0} / \Delta r<1 / \gamma=2.5$, smooth outflows for large outlets, and intermittent flow in between. (c) The relative proportion of time steps $\Delta t$ with a stopped outflow confirms this picture. Our results are quite insensitive to the selected parameters. For illustration, we chose $\beta=3, \gamma=2 / 5, \epsilon=0.01, Q_{\text {in }}=4 / \Delta t$, $v_{0}=\Delta r / \Delta t, \rho_{\max }=1 /(\Delta r)^{2}$, and, in (a), $r_{0}=5 \Delta r$ (jammed conditions).

the balance equation $N(r, t+\Delta t)=N(r, t)+N_{\text {in }}(r, t)-$ $N_{\text {out }}(r, t)$ and $\rho(r, t)=N(r, t) / A(r)$ we get

$$
\begin{aligned}
\rho(r, t+\Delta t)= & \rho(r, t)+\xi_{r}^{+}\left(1-\frac{\rho(r, t)}{\rho_{\max }}\right) \\
& \times \min \left[\left(1+\frac{\Delta r}{r}\right) \rho(r+\Delta r, t), \rho_{\max }\right] \\
& -\xi_{r}^{-}\left(1-\frac{\rho(r-\Delta r, t)}{\rho_{\max }}\right) \\
& \times \min \left[\rho(r, t),\left(1-\frac{\Delta r}{r}\right) \rho_{\max }\right] .
\end{aligned}
$$

Finally, note that the half circle of radius $r_{0}$ around the exit is treated analogously to the shells, but we have to replace the area $A(0)$ by $A_{0}=\pi r_{0}^{2} / 2$ and $-N_{\text {out }}(0, t)$ by $-2 r_{0} \rho_{0}(t) v_{0} \Delta t$ [i.e., the exit width $2 r_{0}$ times the flow $q_{0}(t)=\rho_{0}(t) v_{0}$, if pedestrians can leave with maximum velocity $v_{0}$ into the uncongested space behind the exit]. The resulting equation for the density $\rho_{0}(t)$ in the last (sub)area before passing the bottleneck is

$$
\begin{aligned}
\rho_{0}(t+\Delta t)= & \rho_{0}(t)+\xi_{0}^{+}(t)\left(1-\frac{\rho_{0}(t)}{\rho_{\max }}\right) \\
& \times \min \left[\frac{2 \Delta r}{r_{0}} \rho\left(r_{0}, t\right), \rho_{\max }\right]-\frac{4 \Delta r}{\pi r_{0}} \rho_{0}(t) .
\end{aligned}
$$

The minimum function in Eq. (6) delineates the mergingrelated lack of space and outflow capacity. A similar situation and minimum function occurs in merging flows in urban street and production networks. With $\Delta r=v_{0} \Delta t$, $\rho=\rho(r, t), \xi_{r}=\left(\xi_{r}^{+}+\xi_{r}^{-}\right) / 2$, and $\zeta_{r}=\left(\xi_{r}^{+}-\xi_{r}^{-}\right)(1-$ $\left.\rho / \rho_{\max }\right) / \Delta t$ and for $\rho(r, t) \leq(1-\Delta r / r) \rho_{\max }$, we find the following equation in the limit $\Delta t, \Delta r \rightarrow 0$ :

$$
\frac{\partial \rho}{\partial t}=v_{0}\left(\xi_{r}^{+}-2 \xi_{r} \frac{\rho}{\rho_{\max }}\right) \frac{\partial \rho}{\partial r}+\frac{\rho v_{0} \xi_{r}^{+}}{r}\left(1-\frac{\rho}{\rho_{\max }}\right)+\zeta_{r} \rho .
$$

With the linear velocity-density relation (3), this exactly corresponds to the previous continuity Eq. (1), if $\xi_{r}^{ \pm}=1$, as for small enough densities (see below). Fluctuations $\xi_{r}^{ \pm}<1$, however, allow one to describe a dynamics in which less particles than possible are successful in finding a gap in the next shell because of coordination problems. The random variable $\xi_{r}^{ \pm}$reflects that the microscopic spatial configuration of the particles matters. When the second terms in the minimum functions of Eq. (6) apply, the dynamics is given by the equation

$$
\frac{\partial \rho}{\partial t}+v_{0} \xi_{r}^{-} \frac{\partial \rho}{\partial r}=\frac{v_{0} \xi_{r}^{-}}{r}\left[\rho_{\max }-\rho(r, t)\right]+\zeta_{r} \rho_{\max } .
$$

After averaging over the noise terms $\xi_{r}^{ \pm}$, representing the average of $\xi_{r}^{-}$by $\bar{\xi}$, defining the gap density $\hat{\rho}(r, t)=$ $\rho_{\max }-\rho(r, t)$, and introducing $\hat{V}=v_{0} \bar{\xi}$, this turns into a continuity equation for gap propagation:

$$
\frac{\partial \hat{\rho}}{\partial t}+\frac{\partial(\hat{\rho} \hat{V})}{\partial r}=-\frac{\hat{\rho} \hat{V}(t)}{r} .
$$

Note that gaps propagate with velocity $\hat{V}>0$, i.e., in opposite direction to the particles.

We expect that a switching between gap propagation and particle propagation by the minimum function can account for the intermittent outflows of dense granular flows. Triggered by the randomness of the variable $\xi_{r}^{ \pm}$, the switching mechanism can produce particle avalanches of different sizes. The fluctuations $\xi_{r}^{ \pm}$and their average value $\bar{\xi}$ can be adjusted to experimental or suitable microsimulation results, e.g., to reflect the spatiotemporal fluctuations due to granular force networks. Here, we have instead simulated Eq. (6) with binomially distributed values of $\xi_{r}^{ \pm}$, i.e. $\xi_{r}^{ \pm}=k / N$ with

$$
P(k)=\left(\begin{array}{c}
N \\
k
\end{array}\right) p^{k}(1-p)^{N-k} .
$$

$P(k)$ is the probability that $k \in\{0,1, \ldots, N\}$ of $N=N(r, t)$ particles successfully manage to move forward, where $p=$ $\bar{\xi}$ is the probability of a particle not to be obstructed. We have used the phenomenological specification

$$
p(\rho, r)=\left\{1+\left[\frac{r}{\Delta r}\left(\frac{\rho_{\max }}{\rho}-1\right)^{\beta}+\epsilon\left(\gamma-\frac{\Delta r}{r}\right)\right]^{-1}\right\}^{-1}
$$

if $p>0$; otherwise, $p:=0 .(\beta, \gamma$, and $\epsilon$ are non-negative fit parameters.) This ensures that $p(\rho, r)$ becomes 1 for 
$\rho \rightarrow 0$ or $r \rightarrow \infty$ and $\max [0, \epsilon(\gamma-\Delta r / r)]$ for $\rho \rightarrow \rho_{\max }$. That is, we have complete clogging, if $r_{0} / \Delta r<1 / \gamma \approx$ $5 / 2$, which reflects arching if the outlet $2 r_{0}$ is too small (see Fig. 2). Otherwise, if the density is low or the bottleneck far away, we have $p=1$ corresponding to particles moving at the speed $v=V(\rho)$. In queued areas with $\rho \approx$ $\rho_{\text {max }}$, gaps propagate upstream with velocity $\hat{V}=v_{0} \epsilon(\gamma-$ $\Delta r / r)$.

We have presented a shock wave approach to determine the spatiotemporal density profile in granular bottleneck flows and evacuation scenarios. Generalized to two dimensions allowing one to consider boundary conditions, friction, etc., there is a free-flow regime characterized by forward motion according to $\partial \rho / \partial t+$ $\partial(\rho V) / \partial x=-[\rho V / w(x)] d w(x) / d x$, where $(d w / d x) / w=$ $\left(\partial V_{\perp} / \partial x_{\perp}\right) / V$ replaces $1 / r$ and describes the relative change of locally available width $w$, i.e., the bottleneck effect. $x$ is the coordinate in flow direction and $V>0$ the corresponding speed, while $\perp$ represents the perpendicular direction. If $w(x) \rho>[w(x)+\Delta x d w / d x] \rho_{\max }$, the density after the next step of length $\Delta x$ (where $\Delta x$ is the mean free path or a fit parameter) would exceed the maximum possible density $\rho_{\max }$. Therefore, if the "gap density" $\hat{\rho}(x, t):=\rho_{\max }-\rho(x, t)$ falls below the value $-\rho_{\max } \Delta x d w / d x$, we have instead the equation $\partial \hat{\rho} / \partial t+$ $\partial(\hat{\rho} \hat{V}) / \partial x=-[\hat{\rho} \hat{V} / w(x)] d w(x) / d x$ for gap propagation, where $\hat{V}<0$ is the backward propagation speed. Hence, at bottlenecks we have alternating phases of forward pedestrian motion with speed $V$ and of upstream dissolving pedestrian jams with average speed $\hat{V}$, where $\hat{V}$ and $V$ may fluctuate in space and time.

These formulas are useful for the appropriate dimensioning of exits in order to avoid critical situations in cases of emergency evacuation of people. If the bottleneck is too small (and the desired speed $v_{0}$ of pedestrians too high), one may find intermittent flows close to and behind the bottleneck [Figs. 2(b) and 2(c)]. These are due to the fact that too many "particles" are competing for a confined space. Obviously, not all particles can successfully progress when there are mutual obstructions. This "coordination problem" has been reflected by a fluctuation factor $\xi_{r}^{ \pm}$, the mean value $p(\rho, w /(d w / d x))$ of which drops significantly below 1 if $\rho(r, t) / \rho_{\max }>1+$ $\Delta r(d w / d x) / w(x)$, i.e., if not all particles fit into the reduced space when progressed by a distance $\Delta x$.

The resulting dynamics is related to a stop-and-go phenomenon: In the high-density jam, the velocity is zero, as the particles cannot move. However, jam resolution at the exit causes an upstream moving shock wave, in front of which the density is low. Therefore, particles at the jam front can leave the jam. In fact, if the density in front of the jam is small enough, there is a forward motion of particles filling the low-density area. Altogether, we will have alternating phases of jam resolution and gap filling processes close to the exit, which leads to alternating propagation directions of the jam front. This may also explain the observed alternation in the propagation direction of perturbations in freeway traffic flows [12].

Support by the DFG (No. He2789/7-1) is acknowledged.

[1] Physics of Dry Granular Media, edited by H. J. Herrmann, J.-P. Hovi, and S. Luding (Kluwer Academic, Dordrecht, 1998); H. M. Jaeger and S. R. Nagel, Rev. Mod. Phys. 68, 1259 (1996).

[2] G. H. Ristow and H. J. Herrmann, Phys. Rev. E 50, R5 (1994); K. To, P.-Y. Lai, and H. K. Pak, Phys. Rev. Lett. 86, 71 (2001).

[3] R. Peralta-Fabi, C. Málaga, and R. Rechtman, Europhys. Lett. 45, 76 (1999); D. E. Wolf and P. Grassberger, Friction, Arching, Contact Dynamics (World Scientific, Singapore, 1997).

[4] J.-L. Aider et al., Phys. Rev. E 59, 778 (1999); K.-L. Schick and A. A. Verveen, Nature (London) 251, 599 (1974); G. Peng and H. J. Herrmann, Phys. Rev. E 49, R1796 (1994); O. Moriyama et al., Phys. Rev. Lett. 80, 2833 (1998).

[5] E. Clément et al., in Traffic and Granular Flow '99, edited by D. Helbing, H. J. Herrmann, M. Schreckenberg, and D. E. Wolf (Springer, Berlin, 2000), pp. 457.

[6] D. Helbing et al., Transp. Sci. 39, 1 (2005).

[7] D. Helbing, I. Farkas, and T. Vicsek, Nature (London) 407, 487 (2000).

[8] W. J. Yu et al., Phys. Rev. E 72, 026112 (2005).

[9] A. Kirchner, K. Nishinari, and A. Schadschneider, Phys. Rev. E 67, 056122 (2003).

[10] T. Nagatani, Physica (Amsterdam) 300A, 558 (2001).

[11] See supplementary material for this Letter at www. trafficforum.org/waves.

[12] B. S. Kerner and H. Rehborn, Phys. Rev. E 53, R4275 (1996).

[13] J. Laval and C. Daganzo, ITS Report No. 2004-1, 2004.

[14] D. Helbing, R. Jiang, and M. Treiber, Phys. Rev. E 72, 046130 (2005).

[15] L. P. Kadanoff, Rev. Mod. Phys. 71, 435 (1999).

[16] S. N. Coppersmith et al., Phys. Rev. E 53, 4673 (1996); M. E. Cates et al., Phys. Rev. Lett. 81, 1841 (1998).

[17] Such mechanisms may also change the statistics (e.g., stick-slip friction would cause self-organized criticality with power-law distributed avalanche sizes [4]).

[18] G. B. Whitham, Linear and Nonlinear Waves (Wiley, New York, 1974). 